\title{
Téoros
}

Revue de recherche en tourisme

\section{Le Saint-Laurent, mémoire du pays}

\section{Normand Cazelais}

Volume 6, numéro 2, juillet 1987

Le Saint-Laurent magnétique

URI : https://id.erudit.org/iderudit/1080500ar

DOI : https://doi.org/10.7202/1080500ar

Aller au sommaire du numéro

Éditeur(s)

Université du Québec à Montréal

ISSN

0712-8657 (imprimé)

1923-2705 (numérique)

Découvrir la revue

Citer ce document

Cazelais, N. (1987). Le Saint-Laurent, mémoire du pays. Téoros, 6(2), 3-32. https://doi.org/10.7202/1080500ar d'utilisation que vous pouvez consulter en ligne.

https://apropos.erudit.org/fr/usagers/politique-dutilisation/ 


\title{
En remontant aux sources de la toponymie...
}

\author{
par la Commission de Toponymie du Québec
}

Jusqu'aux dernières années du XVII'siècle, l'unique chemin qui relie Québec à Montréal, et ces villes à la campagne, reste le Saint-Laurent et ses rivières tributaires.

Canots, voiliers, barques à rames ou à voiles sillonnent cette route naturelle et portent d'une ville à l'autre nouvelles et marchandises, parfois avec un retard qui peut atteindre trois semaines quand les elements ne sont pas favorables.

Le spécifique Saint-Laurent apparaît comme toponyme dans des traductions de la narration de Jacques Cartier concernant son voyage de 1535-1536: d'abord dans une traduction en espagnol en 1552 et une autre en italien en 1556. Dans ces traductions, l'appellation baye sainct Laurens, attribuée par Cartier en 1535 à un rentrant de la Côte-Nord, est rendue principalement par grand fleuve de Saint-Laurent. Par la suite, le spécifique Saint-Laurent s"est aussi appliqué au golfe puis au cours d'eau. L'appellation la plus ancienne à avoir été consignée pour caractériser cette entité hydrographique demeure celle mentionnée par Jacques Cartier en 1535, qui est celle de grand fleuve de Hochelaga.

Cependant, ainsi qu'en témoignent certains documents, le nom peut-être le plus connu du fleuve au XVI $\mathrm{I}^{\mathrm{e}}$ siècle fut rivière $d u$ Canada. Par exemple, dans une lettre datée de 1587, Jacques Noël, petit-neveu de Cartier qui lui aussi avait remonté ce cours d'eau au moins jusqu*à la hauteur de Montréal, écrit également rivière de Canada. En 1603, Samuel de Champlain l'a aussi désigné sous ce nom. Il opta par la suite pour grande rivière de sainct Laurens et fleuve sainct Laurens. C'est donc au XVI' ${ }^{e}$ siecle que $I^{\dagger}$ appellation fleuve SaintLaurent a fini par supplanter ses concurrents.

Depuis le $X V T^{*}$ siecle, des segments de cette voie d'eau ont parfois pris des appellations spécifiques. Par exemple, lors de son expédition de 1542-1543, l'explorateur français Jean-François de La Roque de Roberval nomma France Prime la section comprise entre l'ile d'Orléans et la localité de Cap-Rouge. Selon le géographe anglais

"Organiame gouvernemental.
Richard Hakluyt, ce nom de France Prime a été attribué par Roberval en hommage à François Premier, roi de France. Aux $\mathrm{XVII}^{e}$ et XVIII ${ }^{e}$ siècles, le Saint-Laurent, entre les lacs Saint-François et Ontario, a parfois été indiqué sur les cartes sous les noms de rivière des Iroquois et de rivière de Cataracoui. Cette dernière appellation venait du nom amérindien du fort Frontenac, aujourd'hui Kingston en Ontario. Ces

deux toponymes devaient disparaitre et être remplacés par fleuve Saint-Laurent

Rappelons enfin que les nations amérin. diennes ont chacune leurs appellations pour identifier le fleuve. En montagnais, le Saint-Laurent se dit Wepistukujaw Sipo ("rétrécissement"'), en abénaquis Moliantegok ("à la rivière Montréal"), en mohawk Raiatatokenti et Raoteniateara (aucune traduction connue). $f$

\section{Le Saint-Laurent, mémoire du pays}

\section{par Normand Cazelais*}

Le Saint-Laurent est fils de la mer de Champlain et dauphin du golfe de Laflamme; il est héritier du Grand Glacier qui a varlopé en temps d'inhumaine mémoire la table bosselée du Bouclier et laissé, à son retrait, terrasses dans la plaine et plages sur les hauts versants des montérégiennes. Mais pourquoi, oui pourquoi, une légende ne nous ranconte-elle pas que le Saint-Laurent est né d'un déchirement d'amour ou d'un long hozanna?

Ohé, là-bas! m'entendez-vous? L'eau porte-elle ma voix jusqu'à vous? N'étiezvous pas marin sur ce navire, l'Emérillon? Que vous a confie ce fleuve, cette rivière de rivières? Vous qu'il a, les premiers, accueillis à sa cour, en ses vastes eaux, avez-vous pu parcourir son domaine avec lui? L'avez-vous vu, dites-moi, recevoir la Romaine, l'Aguanus, le Richelieu, I'Outaouais, tous ses émissaires, ses vassaux, grands et petits, venus lui rendre compte de ses terres hautes et vierges possessions, venus lui raconter le Midi comme le Septentrion? A-t-ill, dites-moi, calmé les plus nerveux, dompté les rapides et bouillonné a la rencontre du Saguenay, fjord qu'on m'assure être son grand ami? Vous a-t-il permis d'ecouter avec lui bruisser dans le soir la forêt de ses chênes, de ses

*Normand Cazelais, góographe, chroniqueur de tourisme, est membre du comite de rédaction de Téoros et coordonnataur du present numéro. hêtres et érables, de ses ormes, de ses frênes et bouleaux, de toute sa forêt qui se découvre chaque année devant sa douleur automnale?

$D^{*}$ Anticosti à la plaine, le Saint-Laurent est golfe, estuaire et fleuve. Il est l'un, l'autre et les trois. Et bras de mer et sang de la terre. Il est veine battante aux caresses de juillet, il est strate et métal dérobés par le gel, il est nerf tendu entre l'Ancien et le Nouveau Monde. J'y colle l'oreille et entends le cri de l'outarde, l'écho des bris de glace et du grand craquement des ponts effondrés; je retiens mon souffle, je n'entends plus le foc claquer mais seulement gronder les moteurs et mugir les sirènes. Le Saint-Laurent n'a pas tout oublié. D'ailleurs, il n'a rien oubliê, il préfere parfois ne pas se rappeler.

Oui, le Saint-Laurent est mémoire du Québec: il a mémoire de ces rangs développés, perpendiculaires à ses eaux, en longues lanières parallèles; il a mémoire du fronteau, du premier voisin et du trécarré, des seigneuries et de leurs moulins où les barques y menaient le grain, de Sainte-Lucesur-mer, de Métis-Beach et des premières stations balnéaires recherchées des Américains. Il porte encore l'entaille des pre-

(suite a la page 32 ) 


\section{Les risques et les efforts de la linnéenne}

En 1985, la Linnéenne du Québec poursuit son expansion le long des rives du SaintLaurent: après la cogestion à I"A quarium de Québec, la gestion à la halte cötière de pointe Noire, elle soumissionne pour être cogestionnaire à la réserve nationale de faune de Cap-Tourmente qu'elle obtiendra le ler avril 1985 . Le temps qu'il reste aux bénévoles va au dossier des croisières pour en effectuer un bilan. Ainsi, pendant quelques années, la Linnéenne a pris tous les risques de recherche, d'expérimentation et de commercialisation tout en respectant le rốle des bateliers privés; dans un deuxième temps, nous avons partagé les risques avec les bateliers retenus pour un produit touristique donné en réalisant bien que ces derniers tentaient de nous marginaliser, saison après saison. Entre toutes les options examinées, nous en acceptons finalement une nouvelle venant d'un nouveau batelier de Tadoussac qui offre de prendre tous les risques à sa charge en contrepartie d'un support exclusif pour la référence de clientêle et les services d'interprétation. Nous serons donc trois organisations à desservir le public sur lá rive nord pour un marché qui continue à croître: celuì-ci (figure 1) entraine des retombées économiques dans la région de plus de trois millions de dollars.

Le 23 novembre 1985, la Linnéenne reçoit le prix François de B.-Gourdeau, remis annuellement par le ministère du Loisir, de la Chasse et de la Pêche du Québec à un organisme de conservation et elle continue en 1986 sur sa lancée initiale en poursuivant sensiblement les mëmes dossiers terrestres et nautiques. Une année de consolidation où nous n'ajoutons qu'un produit d'importance, soit l'instauration d'une croisière expérimentale comprenant un départ de Tadoussac en voilier et un sêjour à l'île Verte .

Après tant d'efforts, le Saint-Laurent est redevenu un puissant attrait touristique. $f$

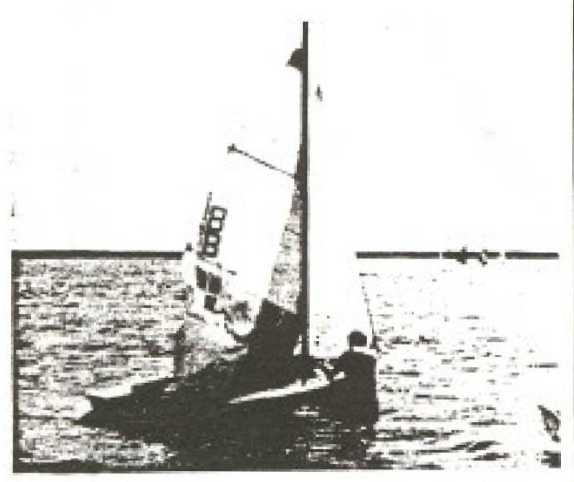

\section{Ribibrence}

(1) Mme Suzanne Nomandeau et M. Rend Roy. furent aussi las autres responsabies du projet.

\section{Le Saint-Laurent} de demain

(suite de la page 25)

voie de faire subir au Vieux-Port. Dans cette ville au cachet européen et historique unique, on opte pour le moderne, les constructions commerciales en hauteur, sans souci d'intégration, sans plan directeur ni consultation publique. Rien, pourtant, ne se démode plus rapidement que le style futuriste!

De Lotbinière à Saint-Jean-Port-Joli, le plan de développement touristique du Paysde-l'Érable propose une série de projets de mise en valeur, notamment à Pointe au Platon sur la côte de Lotbinière, dans le VieuxLévis et le Vieux-Lauzon. Plus en aval, le plan propose de faire de la Côte-du-Sud une zone de destination touristique reposant sur la mise en valeur du parc historique national de Grosse-Ile, le bassin de Montmagny et le reste de l'archipel. Selon Parcs Canada, le projet touristique de Grosse-Ile pourrait générer une affluence de 80000 visiteurs par année.

Dans Charlevoix, le projet de création d'un parc marin à l'embouchure du Saguenay et sur le littoral, la mise en valeur du site de l'ancien chantier naval de Saint-Joseph-dela-Rive, la station de Petite-Rivière-SaintFrançois dont l'intérêt réside en grande partie dans le mariage entre la montagne et le fleuve, sont au nombre des attraits d'avenir.

Les plans de développement touristique des régions du Bas-Saint-Laurent et de la Gaspésie réalisés il $\mathrm{y}$ a quelques années sont en voie de révision. Mentionnons toutefois une étude en cours portant sur la faisabilité d'un centre d'interprétation maritime à Pointe-au-Père. Enfin, sur la Côte-Nord, les régions de Manicouagan et Duplessis préparent leurs plans respectifs.

\section{De la grande visite}

L'industrie et le marché mondial des croisières touristiques sont concentrés aux

\section{Le Saint-Laurent mémoire du pays}

(suite de la page 4)

miers chenaux qui ont favorisé en ses berges ports, industries et urbanisation.

A Verchères et Lavaltrie. Donnaconna et Deschaillons, Kamouraska et Cap-àl'Aigle, des gens regardaient l'autre rive sans jamais y aller, ou attendaient l'hiver; aux Ilets-Caribou et Cap-Chat, à Magpie et Cloridorme, il y a des gens qui ne voient jamais l'autre rive. Mais d'Alençon à SaintJoseph-de-Sorel, de Deschambault à Lotbinière, de Québec à Lévis, des Escoumins à Trois-Pistoles, de Matane à Godbout, i] y a chaque jour le réseau sans cesse tressé des traversiers.
États-Unis. Les grandes compagnies maritimes, considérant le quadrilatère que forme le continent nord-américain, ont d'abord exploité à fond le sud-est, les Caraibes, puis le sud-ouest, la Riviera mexicaine. Ces dernières années, le nordouest. I Alaska, a connu un essor touristique remarquable. Reste le nord-est, constitué de la Nouvelle-Angleterre. des Maritimes et du Saint-Laurent (c'est-ă-dire le lieu des anciennes croisières).

Attendons-nous à voir, au cours des prochaines années, la visite de plus en plus fréquente des magnifiques navires de la nouvelle armada de cette industrie en pleine expansion. A eux seuls, ces navires de grande classe ${ }^{(9)}$ constituent une attraction. $f$

\section{Réfárences et notes}

(1) Document rendu public en mars 1987 par le MLCP $220 \mathrm{p}$

(2) A noter que de tels organismes se sont développés alleurs en fonction de cours d' eau aux dimen. sions plus réduites.

(3) II y en a certes trop peu. Entre Montreal et Québec, cela peut śsexpliquer en partie par le manque đopportunite et d'accessibilite mais plus en aval, le phenomene se justifie moins bien.

(4) A surveiller: les rebsultats de la Conférence internationale des maires du Saint-Launont ot des Grand: Lacs la Quebec les 28 et 29 mai 18d7). Cette conférence bi-nationale a étudié les avenues nouvel. les pour promouwoir le commerce et le tourisme a l'intérieur du systeme maritime SaintLaurent/Grands Lacs.

(5) Le tourisme à Montreal, bilan et perspectives de développement, OPDO, 1986, 267 p.

(6) Marsan, Jean-Claude, Montribal, une esquisse du futur, IORC, 1983.

(7) Association touristique regionale du Coeur du Quebec, Plen de developpement touristique régional du Coeur du Qubbec, septembre 1986.

(8) Ibidem, p. B6

(91) Le Veracruz, gue I'on connait bien, est classe deux 6toiles (classification de Berlitz). A titre de com. paraison, les navires qui depuis peu sillonnent le Saint-Laurent, tels le Royal Viking Sea, le Rova Viking Sky at POceen Princess, sont claseds cing etoiles, te Stella Solaris at is Royal Odvssey, qua tre thoiles. La Sitmar Cruises Line projette de venir a Montréal en 1988. Ses naviras sont clased's quatre étoiles.
D'Anticosti à la plaine, le Saint-Laurent habille le Québec: corsage entrouvert, il laisse, sous des bordures de dentelle, soupçonner des charmes épanouis, imaginer des formes pleines et généreuses, des grains d'une peau tressaillante qui s'appellent I'Islet, Gaspésie, Côte-Nord et Mauricie. Désirer des abandons. Il pare sa gorge d'une riche rivière d'îles emprisonnées et libérées tout à la fois, sculptées, pressées, caressées.

Quand le Saint-Laurent endort ses iles à l'automne, je suis le père Didace qui pousse, du long batton, son bateau entre celles de Sorel. Je vais, derrière cet homme debout, à la rencontre de notre commun ancêtre. $f$ 